\title{
Internal Anisotropy of Collision Cascades
}

\author{
F. Kun*, G. Bardos \\ Department of Theoretical Physics, Kossuth Lajos University P.O. Box 5, H-4010 Debrecen, Hungary
}

(November 19, 2018)

\begin{abstract}
It is shown that for collision cascades the global fractal dimension cannot give an adequate description of the geometrical structure because it is insensitive to the internal anisotropy of the object arising from the directionality of cascade branches. In order to give a more elaborate description of the cascade we introduce an angular correlation function, which takes into account the direction of the local growth of the branches of the cascades. It is demonstrated that the angular correlation function gives a quantitative description of the directionality and the interrelation of branches of the cascade. The power law decay of the angular correlation is evidenced and characterized by an exponent $\beta$ and an angular correlation length $R_{a}$ different from the radius of gyration $R$. It is demonstrated that the overlapping of subcascades has a strong effect on the angular correlation.
\end{abstract}

PACS numbers: 47.53.+n, 47.54.+r, 61.43.+Hv

\section{MOTIVATIONS}

Collision cascades develop in condensed matter as a consequence of irradiation with energetic beams of particles. The bombarding particles transfer their kinetic energy in series of collisions with the target atoms and the energized, recoiling atoms generate further recoils in their own slowing-down process. The result of this energy sharing process is a collision cascade. The cascade can also be considered as a far-from equilibrium process leading to structure formation in the solid target. Recently the geometrical structure of collision cascades has been analyzed by means of analytical approaches and Monte Carlo simulations concentrating on the possible fractal and multifractal aspects. These investigations have been extended to the study of the self-similarity properties of the cascade [1 3], to the determination of its fractal dimension and multifractal spectra for different interaction potentials [4 9], and they gave an insight into the cascade-subcascade transition and into the spike creation [1.2,2,9].

Far from-equilibrium processes often create complex geometrical structures which exhibit fractal properties characterized by the fractal dimension $D$ [10]. However, the fractal dimension is a global property of a cluster of particles, it does not provide an insight into the structural details of the object. It has been demonstrated in the case of Diffusion Limited Aggregation (DLA) that large branching structures may be internally anisotropic. This anisotropy results in a tangential correlation different from the radial one 11] and it shows up in the behavior of the three point correlation function as well [12.

The internal structure of collision cascades, the cor- relation of the vacancies, the interrelation of branches and the directionality of ion tracks can have an important impact on several physical processes, e.g. ion-beam mixing [13], radiation enhanced diffusion [13, 14, electric and optical properties of irradiated polymeric materials [15, 16], the development of mechanical stress due to ion bombardment and crack propagation in the solid from the irradiated zone [17].

In the case of diffusion, for example, the correlation of vacancies due to the directionality of the cascade branches can enhance the diffusion process in the irradiated zone. Inside the cascade the path of the displaced atoms can be viewed as conduit, forming a labyrinth in the damaged material [14]. Thus, we are led to de Gennes's model of "termite diffusion", when the diffusing particles (termites) meet the conduit-labyrinth, they diffuse much more quickly and over much greater distances than they do in the bulk 18 .

Further motivations to study the directionality of ion tracks in a solid comes from the earlier studies of system of particles interacting via elastic collisions. A system where many particles scatter, interacting elastically with each other, may be regarded as a model of gas. In such a system each particle is scattered successively, so it walks almost randomly. Although the velocity autocorrelation of such a particle was believed to decay exponentially, Alder and Wainwright discovered a long time tail by computer simulation [19,20]:

$$
\langle\vec{v}(t) \cdot \vec{v}(0)\rangle \sim t^{-d / 2}, \quad d \geq 2,
$$

where $d$ is the dimension of the embedding space. Long time tails of random walks are also found in a much simpler system called the Lorentz gas model where a classical particle is scattered elastically off randomly located fix scatterers. (This model was originally introduced as a

Electronic address: feri@dtp.atomki.hu 
model of electron motion in a metal.) In the case where the scatterers form a regular lattice, it is proved that the velocity autocorrelation has exponential decay. On the contrary if the scatterers are distributed at random forming a fractal with dimension $D$ there is a long time tail for the negative values of the scalar product of the velocities [21]:

$$
\langle\vec{v}(t) \cdot \vec{v}(0)\rangle \sim-t^{-\left(\frac{d-D}{2}+1\right)} .
$$

Hence the memory effect in the directionality seems to be a common feature of the motion of particles scattered randomly.

Based on the above motivations, in the present paper we want to obtain a more elaborate picture of collision cascades gaining information about the internal branching morphology. The computer simulations have the advantage that one can monitor such quantities which are hard to measure or are not measurable but they can help the understanding of a physical process. In the study of ion-solid interaction in certain cases not only the distribution of the vacancies is of interest but even the path of the moving particles. Obviously, the study of the ions's paths includes the vacancies as well, the position of the vacancies are the branching points in the cascade tree. Thus in the present study not only the vacancies but all the collisional points are included in the analysis of the cascade regardless of the vacancy creation, that can easily be done by means of computer simulations. We performed Monte Carlo simulations of self ion collision cascades in two and three dimensions. For the three-dimensional simulations the scattering cross section $d \sigma=C(m) E^{-m} T^{-1-m} d T$ belonging to the inverse power law potential $V(r) \sim r^{-1 / m}$ was applied. Here $E$ is the energy of the bombarding particle, $T$ is the energy transferred to the target atom in the scattering process and $m$ denotes the parameter of the interaction potential $0<m \leq 1$. In two dimensions we established a toy model with the scattering cross section $d \sigma=K E^{-\frac{m}{2}} T^{-1-\frac{m}{2}} d T$ [7]. A two dimensional cascade does not have experimental relevance but it can help to reveal the role of the embedding dimension $d$ in the formation of the cascade structure. For further details about computer simulation of ion-solid interaction see also Ref. [22].

In the following, at first the structure of cascades generated by Monte Carlo simulations is analyzed in terms of the density-density correlation function. Then an angular correlation function is introduced based on the direction of the local growth of the cascade branches. It is shown that the angular correlation function gives a quantitative description of the directionality and the interrelation of branches of cascades, furthermore, the role of the intersection of branches is enlightened.

\section{CORRELATION FUNCTIONS}

The usual way of studying the structure of an object composed of $N$ particles in the $d$ dimensional embedding space is in terms of the density-density correlation function $C(r)$ :

$$
C(r)=\frac{1}{N r^{d-1} \Omega_{d} \delta r} \sum_{r-\frac{\delta r}{2}<\left|\vec{r}_{i}-\vec{r}_{j}\right|<r+\frac{\delta r}{2}} \rho\left(\vec{r}_{i}\right) \rho\left(\vec{r}_{j}\right),
$$

where $\rho(\vec{r})$ is 1 if there is particle at $\vec{r}$ and zero otherwise. In our case these particles are the points where collisions occurred in the target material during the evolution of the cascade. $\Omega_{d}$ denotes the solid angle in $d$ dimension. For structures of finite size, like collision cascades, the two point density-density correlation function depends on the overall size of the structure described by a characteristic length $R$ as well as the internal length $r$. In this case the correlation function can be written as $C(r, R)$. Here $C(r, R)$ represents the correlation function averaged over a large number of cascades of the same size $R$. The characteristic macroscopic length of the cascade is the radius of gyration $R$ defined as the average distance of the particles:

$$
R^{2}=\frac{1}{N^{2}} \sum_{i, j}\left|\vec{r}_{i}-\vec{r}_{j}\right|^{2} \sim \frac{\Omega_{d}}{N} \int d r r^{d+1} C(r)
$$

$C(r, R)$ is normalized by the total number of particles $N$ :

$$
N=\Omega_{d} \int d r r^{d-1} C(r)
$$

Assuming that $C(r, R)$ is a homogeneous function of its variables results in the scaling form:

$$
C(r, R)=r^{-\alpha} g(x), \quad x=\frac{r}{R},
$$

where $g(x)$ is constant for $x<<1$ and $g(x) \sim e^{-x}$ for $x>1$. The function $g(x)$ is called scaling function and the exponent $\alpha$ is the scaling exponent. The fractal dimension $D$ of the object is defined through the behavior of the total number of particles $N$ as a function of the radius of gyration $R$, i.e. $N \sim R^{D}$. From Eqs. (5, 6) it follows that $\alpha=d-D$. If Eq. (6) provides a valid description of the geometric scaling properties then plots of $r^{\alpha} C(r, R)$ vs. $r / R$ for structures of different sizes will fall on a common curve (on the scaling function $g(x)$ ). This data collapse provides a reliable way of measuring the scaling exponent $\alpha$ (and thus the fractal dimension $D$ ) by varying the value of $\alpha$ until the best collapse is obtained. Representative examples of the density-density correlation function and its data collapse analysis can be seen in Figs. 1, 2 for the $2 d$ and $3 d$ models. The excellent collapse obtained with ten different system sizes proves the validity of the scaling ansatz of Eq. (6) for both model systems. 
However, this way of description based on $C(r, R)$ cannot reveal anything about the possible internal anisotropy because the density-density correlation function is insensitive to the structural details. In order to obtain information about the branching structure we have to take into account some dynamical features of the growth of the cascade. For this purpose we assign to each particle, i.e. to each point of collision, with position $\vec{r}_{i}$ the unit vector $\vec{p}\left(\vec{r}_{i}\right)$ of the linear momentum of the scattered particle. If there is vacancy creation in a collision the unit vector of the recoiled particle appears at the position of its first collision. With this prescription one and only one unit vector is assigned to each point of the object studied. The $\vec{p}\left(\vec{r}_{i}\right)$ vector characterizes the direction of the local growth of the corresponding branch of the cascade tree at position $\vec{r}_{i}$ during the cascade evolution. Fig. 3 shows the unit vectors $\vec{p}$ attached to the particles. Using the vector field $\vec{p}\left(\vec{r}_{i}\right)$ an angular correlation function $C_{a}(r, R)$ can be introduced with the following definition:

$$
C_{a}(r)=\frac{1}{N r^{d-1} \Omega_{d} \delta r} \sum_{r-\frac{\delta r}{2}<\left|\vec{r}_{i}-\vec{r}_{j}\right|<r+\frac{\delta r}{2}} \vec{p}\left(\vec{r}_{i}\right) \cdot \vec{p}\left(\vec{r}_{j}\right)
$$

The form of this equation is similar to Eq. (3) but in the summation the scalar product of the $\vec{p}\left(\vec{r}_{i}\right)$ vectors is used instead of the product of the one particle densities $\rho\left(\vec{r}_{i}\right)$.

Along a given branch of the cascade the vectors $\vec{p}\left(\vec{r}_{i}\right)$ are correlated in the sense that they are almost parallel to each other hence the scalar product in (7) has values close to 1 . Subbranches appear in the cascade tree as a result of the recoiled particles. In Fig. 3 it can be observed that a high energy recoil can give rise even to an extended subcascade the branches of which grow independently of the other parts of the cascade, the other subcascades. This implies that the average value of the scalar product of vectors $\vec{p}\left(\vec{r}_{i}\right)$ belonging to different subcascades is close to zero or it can even be negative. In this sense the angular correlation function $C_{a}(r, R)$ can measure the directionality of cascade branches with respect to each other. Representative examples of the absolute value of the angular correlation function $\left|C_{a}(r, R)\right|$ of cascades generated in the $2 d$ model are shown in Fig. A. Fig. Ga presents a comparison of the angular and the densitydensity correlation functions belonging to the same system and Fig. $\llbracket p$ shows $\left|C_{a}(r, R)\right|$ for different system sizes $R$. It can be observed that for a certain length range $C_{a}(r, R)$ decays according to a power law with an exponent significantly different from the exponent describing the algebraic decay of $C(r, R)$. At a certain value of $r$ the angular correlation function becomes negative and it goes to zero through negative values at long distances. Let's denote $R_{a}$ the characteristic length where $C_{a}(r, R)$ becomes negative. Increasing the system size $R$ in Fig $\Phi b$ $R_{a}$ is also increasing. The negative part of $C_{a}(r, R)$ has a local minimum (i.e. $\left|C_{a}(r, R)\right|$ has a local maximum) and it goes to zero exponentially due to the finite size cutoff.

Based on the above observations, the same form of scaling ansatz can be assumed for $C_{a}(r, R)$ as for $C(r, R)$, i.e. Eq. (6):

$$
C_{a}(r, R)=r^{-\alpha_{a}} f(x), \quad x=\frac{r}{R},
$$

where $f(x)$ denotes the scaling function and $\alpha_{a}$ the scaling exponent belonging to $C_{a}(r, R)$. At small distances $C_{a}(r, R)$ approaches $C(r, R)$ thus part of the power law decay of $C_{a}(r, R)$ can be attributed to the power law decay of $C(r, R)$, therefore the exponent $\beta$ characterizing the angular correlation is the difference of $\alpha_{a}$ and $\alpha$ :

$$
\frac{C_{a}(r, R)}{C(r, R)} \sim r^{-\beta}, \quad \beta=\alpha_{a}-\alpha
$$

Finally, the internal anisotropy of cascades is determined by two quantities, by the characteristic length $R_{a}$ where $C_{a}(r, R)$ drops to negative values and the exponent $\beta$ describing the speed of the decay of $C_{a}(r, R)$.

In the next section we present the results of the analysis of systematic Monte Carlo simulations in terms of the correlation functions introduced above, and we try to give a simple interpretation of the results obtained.

\section{RESULTS AND DISCUSSION}

In a recent publication (see Ref. [7]) it has been demonstrated that the parameter $m$ of the interaction potential plays the role of a control parameter from the viewpoint of the cascade geometry. For decreasing $m$ a structural transition takes place in the cascade from an open branching structure to a space filling one. When the upper critical dimension $d_{u}$ of the cascade exceeds the dimension of the embedding space $d$, so called geometrical correlations arise in the cascade due to the intersection of different branches and the overlapping of subcascades. These geometrical correlations lead to a fractal dimension $D(m)$ different from the self similarity dimension $D_{o}(m)$ for $m \leq 1$ and $m \leq \frac{1}{3}$ in the $2 d$ and $3 d$ models, respectively [7]. The question naturally arises how the intersection of branches influences the behavior of the angular correlation function introduced.

The above analysis in terms of the density-density and angular correlation functions was performed for cascades in a wide range of the parameter $m$ of the potential ( in the same range as in Ref. [7]) for both model systems. The scaling exponents $\alpha, \alpha_{a}$ and the characteristic length $R_{a}$ were obtained by means of the data collapse method. Since the results concerning to the fractal dimension of cascades have already been discussed in Ref. [7] here we restrict ourself to the discussion of the properties of the angular correlation function. 
Fig. 5 shows representative examples of the data collapse analysis of the angular correlation function in the $2 d$ and $3 d$ models. It can be observed that $C_{a}(r, R)$ exhibits power law behavior for more than one order of magnitude in length. The good quality of the collapse obtained demonstrates the validity of the scaling ansatz Eq. (8). Note, that the positive and negative parts of $C_{a}(r, R)$ have the same scaling properties. The power law behavior of $C_{a}(r, R)$ expresses the fact that there is long range angular correlation inside the cascade, which manifests in the directionality of the branches. The negative regime of $C_{a}(r, R)$ can be interpreted as anti-correlation of the local growth directions at large distances. This implies that the angle of the unit vectors $\vec{p}\left(\vec{r}_{i}\right), \vec{p}\left(\vec{r}_{j}\right)$ tends to be larger than $\frac{\pi}{2}$ for $\left|\vec{r}_{i}-\vec{r}_{j}\right|>R_{a}$. The characteristic length $R_{a}$ where $C_{a}(r, R)$ drops to negative values can be considered as an angular correlation length. Let us denote $\gamma$ the ratio of the angular correlation length $R_{a}$ to the radius of gyration $R$.

From the scaling analysis, the exponent $\beta$ characterizing the decay of $C_{a}(r, R)$ and the ratio $\gamma$ of the angular correlation length $R_{a}$ to the radius of gyration $R$ can be determined precisely. From the simulations it was found that $\gamma$ is between 0.7 and 0.8 for both model systems and within the accuracy of the calculations we could not reveal any systematic dependence of $\gamma$ on the parameter of the potential $m$.

Fig. 6 presents the values of $\beta$ as a function of $m$ for $2 d$ and $3 d$. It is important to note that small value of $\beta$ corresponds to slow decay of the directionality, i.e. to long range angular correlation, while increasing $\beta$ implies the weakening of the angular correlation. In Fig. 6 one can observe that in $3 d$ two different regimes of $\beta$ can be distinguished. If $m \geq 1 / 3$, when no overlap occurs in the cascade, $\beta$ practically coincides with the self similarity dimension $D_{o}$. In this region $\beta$ is increasing for decreasing $m$. When $m$ is close to one (Coulomb-scattering) the probability of creating a high energy recoil is rather small, therefore dense subcascades cannot occur, the cascade is strongly directed giving rise to a small value of $\beta$. Decreasing $m$ gives rise to an increasing number of subcascades growing independently, which results in weakening of the directionality order. This is expressed by the increasing value of $\beta$. At $m=1 / 3$ the self similarity dimension $D_{o}$ and $\beta$ reaches $d / 2$, half of the dimension of the embedding space. For $m<1 / 3$ when the overlap of the branches dominates the cascade structure, $\beta$ remains practically independent of $m, \beta=d / 2$. But this does not entails that the density-density correlation function $C(r, R)$ and the angular correlation function $C_{a}(r, R)$ are independent of $m$. Their exponents $\alpha_{a}$ and $\alpha$ are decreasing with decreasing $m$ but in such a way that their difference remains constant:

$$
\beta=\alpha_{a}-\alpha=\frac{d}{2} .
$$

This argument is also supported by the two dimensional simulations (see Fig. 6a). It has been shown in Ref. [7] that in the two dimensional model the overlap effect, the intersection of branches is always present, in the whole region of $m$. In Fig. 6 $6 a$ it can be seen that $\beta$ is equal to 1 , i.e. $\beta=d / 2$, as it can be expected from the $3 d$ case, see Fig. $6 b$. The result that $\beta=\frac{d}{2}$ throughout the overlapping regime demonstrates that the $\vec{p}\left(\vec{r}_{i}\right)$ vector field does not become isotropic even for high degree of overlap, i.e. for small values of $m$. In the system there is always a preferred direction, namely, the initial direction of the bombarding particle. This also manifests in the overall shape of the cascade. The overall shape is not spherical but it is always elongated along the initial direction of the bombarding particle.

It has been mentioned in the introductory part that the internal anisotropy of DLA clusters was studied by means of the tangential and the three-point correlation functions, which are essentially special type of densitydensity correlation functions. It is important to mention that, beside these techniques, Meakin introduced an angular correlation function of the type of Eq. (7) [23. He assigned a so-called bond vector to each particle in the aggregate. The bond vector $\vec{b}$ is a unit vector pointing in the direction of the bond, which is formed when a new particle is added to the growing cluster. Using the $\vec{b}$ vectors an angular correlation function could be defined. $\beta=0.31$ was found in two dimensional off-lattice simulations 23] that evidenced the slow decay of the directionality of the cluster branches in DLA processes.

Collision cascades have the advantage that the structure formation can be easily controlled by varying the parameter of the interaction potential $m$. This gives us a rich spectrum of possibilities to study the internal anisotropy resulted from the directionality of the branches of the object and it makes also possible to gain information about the role of the intersection of branches.

\section{CONCLUSIONS}

In the present paper we studied the internal anisotropy of collision cascades arising from the branching structure. It was demonstrated that the global fractal dimension cannot account for the internal details of the structure. To give a more elaborate description of the cascade it was necessary to introduce an angular correlation function, which takes into account the direction of the local growth of the cascade branches. With the help of the angular correlation function we could quantify the anisotropy, which manifest in the directionality of the branches. The power law decay of the angular correlation was evidenced and characterized by an exponent $\beta$ and an angular correlation length $R_{a}$ different from the radius of gyration $R$. The overlapping of subcascades has a strong effect on the angular correlation. In the 
absence of overlap $\beta$ coincides with the self similarity dimension, while in the presence of overlap $\beta$ is constant, i.e. $\beta=d / 2$. It is interesting to note that in the presence of overlap solely the dimension of the embedding space determines the speed of the decay of the directionality order. We argued that this internal anisotropy of growth directions shows up in the overall shape of the cascade as well.

\section{ACKNOWLEDGMENT}

The authors are very grateful to K. Sailer and Zs. Gulácsi for the valuable discussions. This work was supported by OTKA T-023844.

[1] Y. T. Cheng, M. A. Nicolet and W. L. Johnson, Phys. Rev. Lett. 58, 2083 (1987).

[2] Y. T. Cheng, in: Materials Modification by High-Fluence Ion Beams, 191-203 (Kluwer Academic Publishers) 1989.

[3] A. G. Wagh, S. K. Gupta, Phys. Lett. A 146, 511 (1990).

[4] K. B. Winterbon, H. M. Urbassek, P. Sigmund and A. Gras-Marti, Physica Scripta 36, 689 (1987).

[5] J. C. Moreno-Marin, U. Conrad, H. M. Urbassek, A. Gras-Marti, Nucl. Instr. and Meth. in Phys. Res. B 48, 404 (1990).
[6] F. Kun, G. Bardos, Phys. Rev. E 50, 2630 (1994).

[7] F. Kun, G. Bardos, Phys. Rev. E 55, 1508 (1997).

[8] R. P. Webb, A. Grass-Marti, I. A. Wilson, D. E. Harrison, E. Louis and F. Guinea, Phys. Lett. A 126, 136 (1987).

[9] F. Rossi, D. M. Parkin, M. Nastasi, J. Matter. Res. Vol. 4., No1 (1989).

[10] Fractals and Disordered Systems, edited by A. Bunde, S. Havlin (Springer - Verlag, Berlin, 1991).

[11] P. Meakin, T. Vicsek, Phys. Rev. A 32, 685 (1985).

[12] T. C. Halsey and P. Meakin, Phys. Rev. A 32, 2546 (1985).

[13] Y. T. Cheng, Materials Science Reports 5, 45 (1990).

[14] G. O. Williams, V. A. Singh, J. W. Corbett, Phys. Stat. Sol. (b) $144 \mathrm{~K} 1$ (1987).

[15] L. Calcagno, G. Compagnini, and G. Foti, Phys. Rev. B 46, 10573 (1992).

[16] L. Calcagno and G. Foti,Nucl. Instr. and Meth. in Phys. Res. B 59/60, 1153 (1991).

[17] E. Louis, A. Gras-Marti, F. Guinea, Nucl. Instr. and Meth. in Phys. Res. B 18, 402 (1987).

[18] P. G. de Gennes, J. Physique 41, C-3, 17 (1980).

[19] B. J. Alder and T. E. Wainwright, Phys. Rev. Lett. 18, 988 (1967).

[20] B. J. Alder and T. E. Wainwright, Phys. Rev. A 1, 18 (1970).

[21] H. Takayasu and K. Hiramarsu, Phys. Rev. Lett. 53, 633 (1984).

[22] Computer simulation of ion - solid interactions, W. Eckstein, (Springer - Verlag, Berlin, 1991).

[23] P. Meakin, J. Phys. A: Math. Gen. 18, L661-665 (1985).

\section{FIGURES}
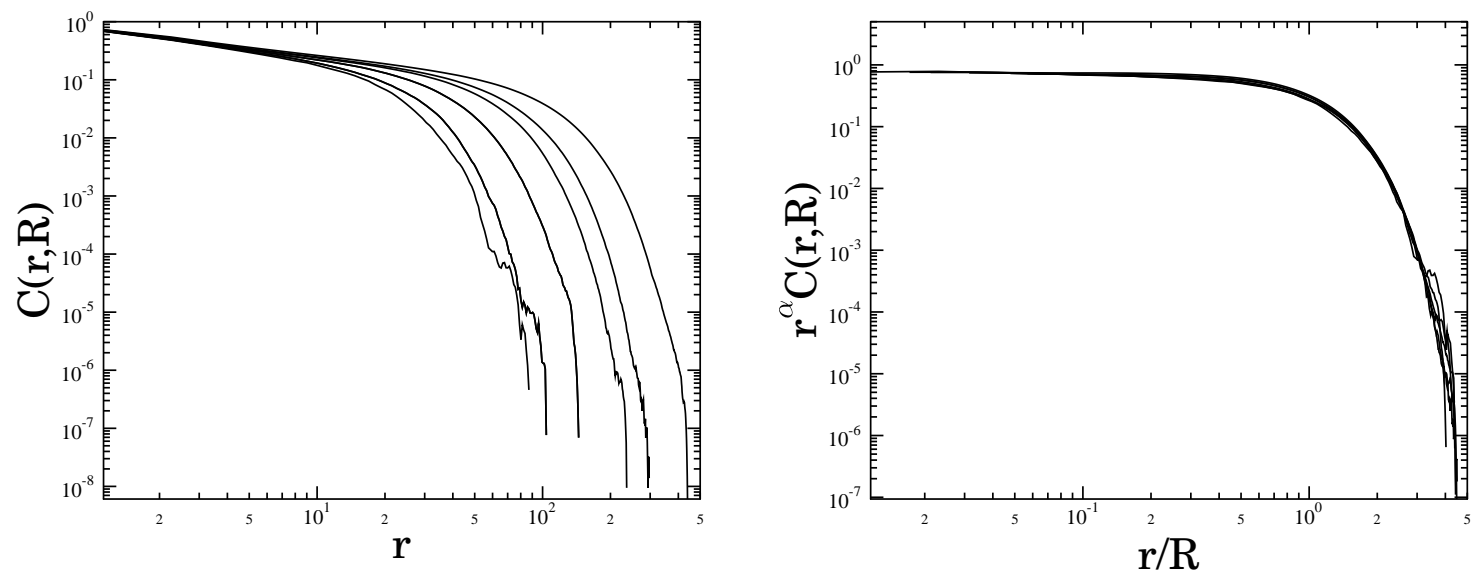

FIG. 1. The density-density correlation function for system sizes from $N=400$ to $N=4000$ in the $2 d$ model. The parameter $m$ of the scattering cross section was chosen to be $m=0.7$. The data collapse analysis was performed with ten different curves. 

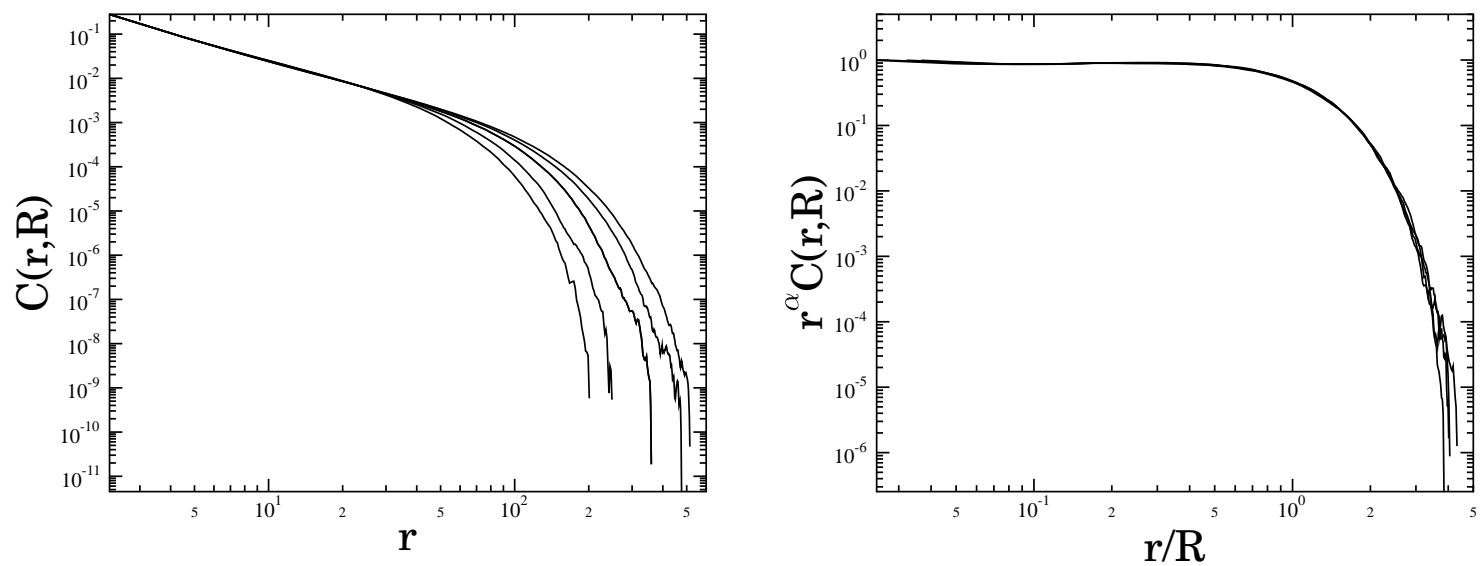

FIG. 2. The density-density correlation function for system sizes from $N=800$ to $N=5000$ in the $3 d$ model. The parameter $m$ of the scattering cross section was chosen to be $m=0.4$. The data collapse analysis was performed with ten different curves.

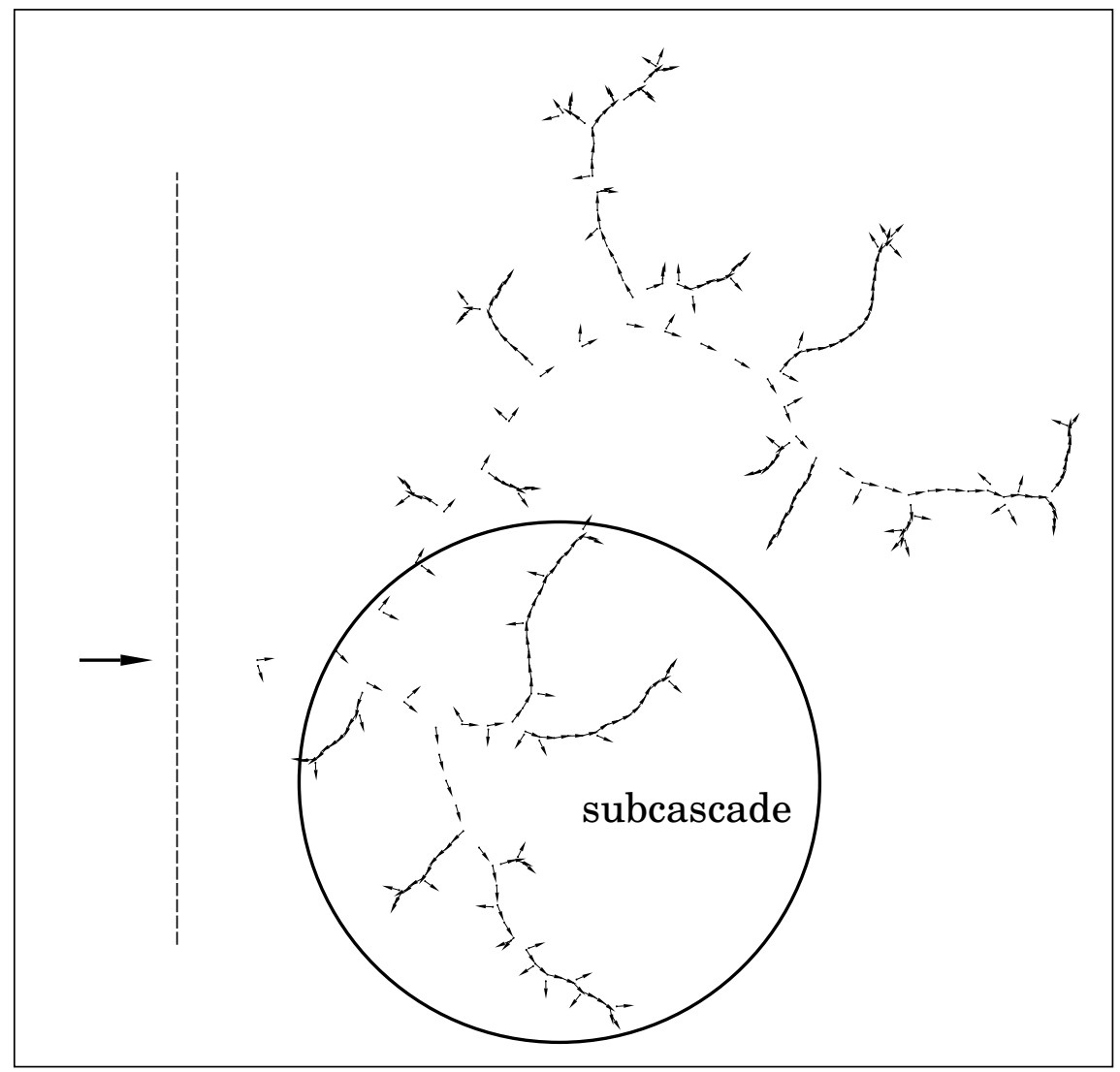

FIG. 3. Collision cascade generated in $2 d$. The arrows represent the unit vector $\vec{p}\left(\vec{r}_{i}\right)$ of the linear momentum of the scattered particles after the collision. $m$ has the same value as in Fig. 1. The circle indicates an extended subcascade resulted from a high energy recoil. The surface of the solid and the direction of the penetration of the bombarding particle are also shown. 

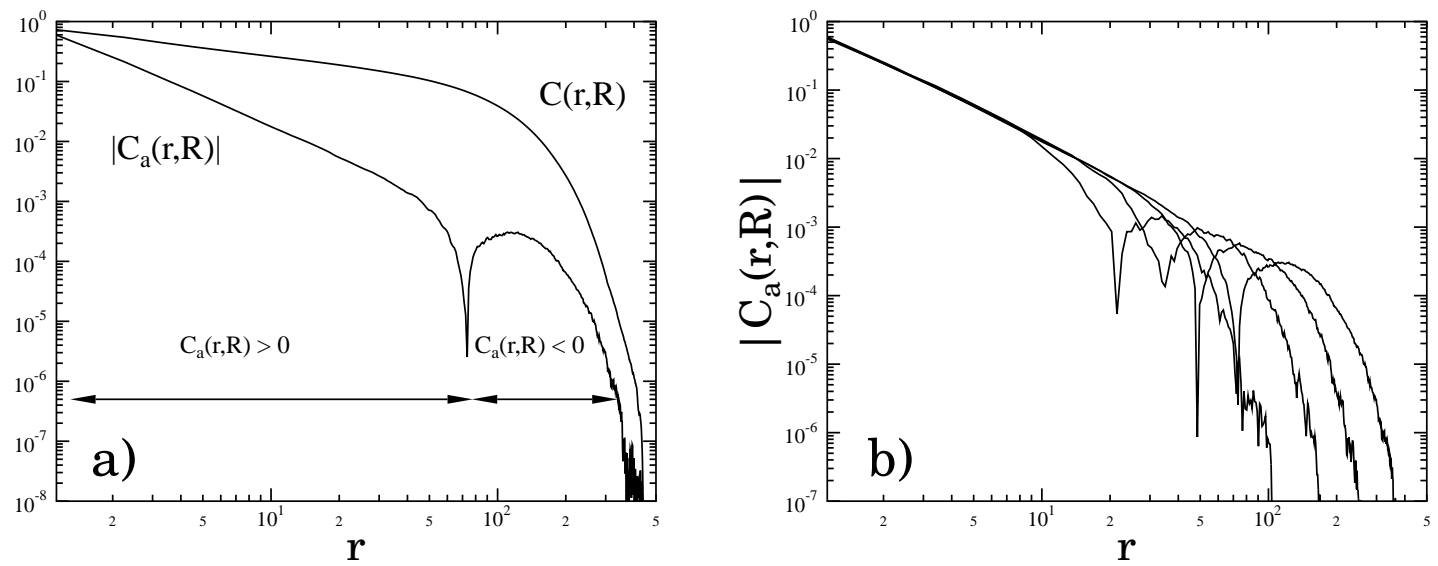

FIG. 4. Representative examples of the behaviour of the angular correlation function $C_{a}(r, R)$ in the same system as in Fig. 11. (a) Comparison of the absolute value of the angular correlation function $\left|C_{a}(r, R)\right|$ and the density-density correlation function $C(r, R)$ belonging to the same system size $R,(b)\left|C_{a}(r, R)\right|$ for different system sizes.
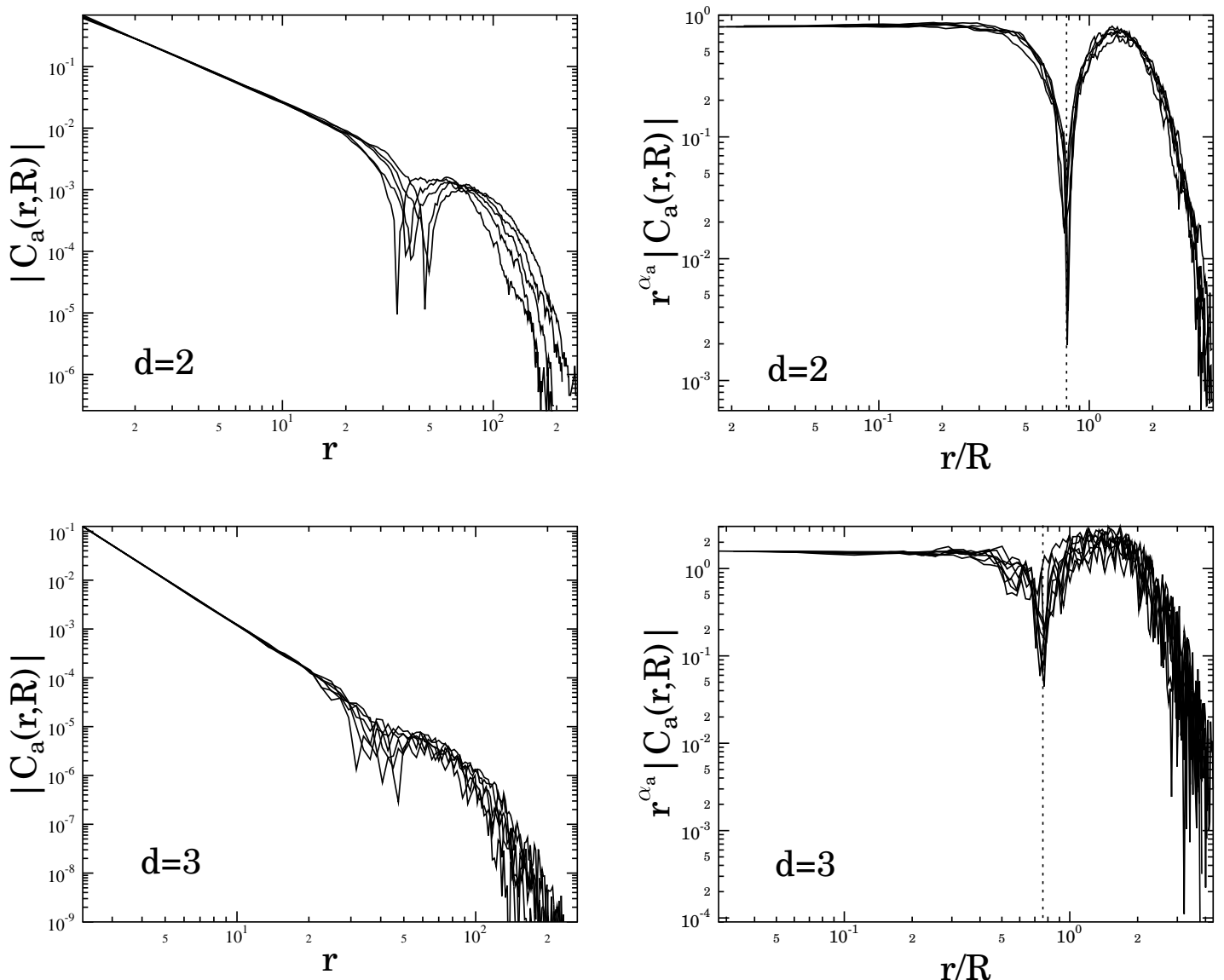

FIG. 5. Data collapse analyzis of the angular correlation function in the $2 d$ and $3 d$ models. One can observe the good quality of the collapse, which demonstrates the validity of the scaling ansatz Eq. (8). The parameter $m$ of the scattering cross section was chosen to be $m=0.7$ and $m=0.4$ in $2 d$ and $3 d$, respectively. The value of $\gamma=R_{a} / R$ is indicated by dotted lines in the figures, it is 0.78 and 0.76 in $2 d$ and $3 d$. 

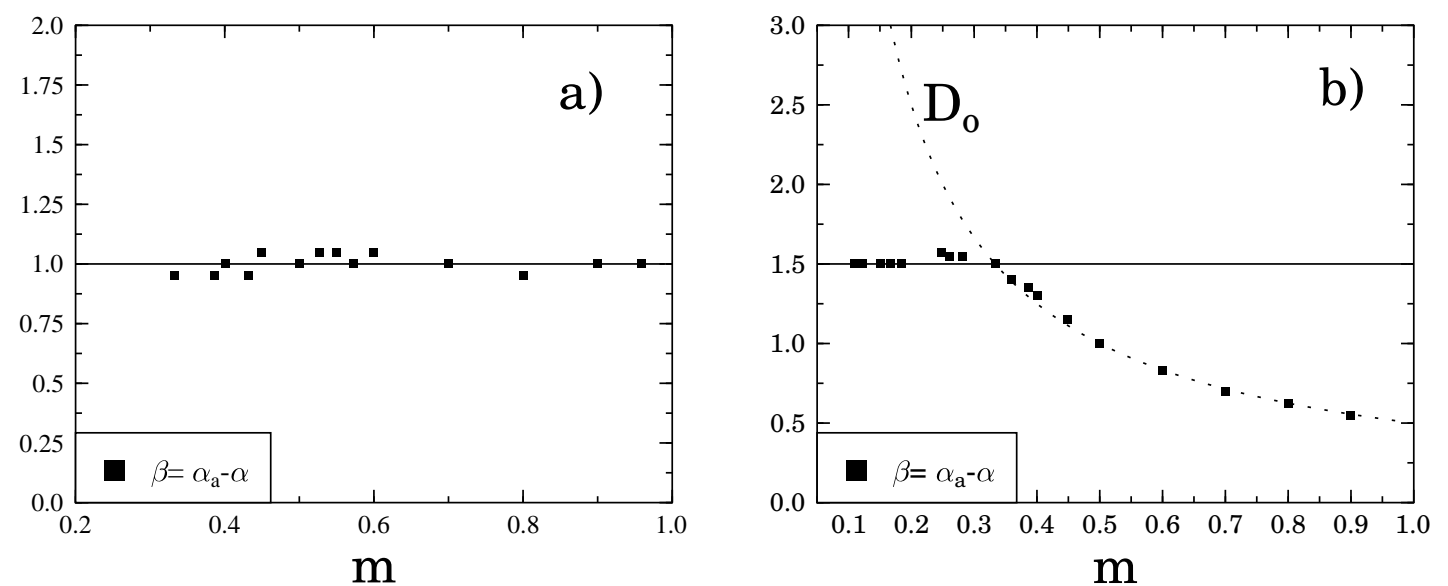

FIG. 6. The exponent $\beta=\alpha_{a}-\alpha$ characterizing the decay of the angular correlation function $C_{a}(r, R)(a)$ for $2 d$ and $(b)$ for $3 d$. In the $3 d$ case the curve of the self similarity dimension is also shown. The horizontal solid lines indicate the value of $d / 2$. 